\title{
Visual Hierarchy and Viewing Behavior: An Eye Tracking Study
}

\author{
Soussan Djamasbi ${ }^{1}$, Marisa Siegel ${ }^{2}$, and Tom Tullis ${ }^{2}$ \\ ${ }^{1}$ Worcester Polytechnic Institute, School of Business, 100 Institute Road, \\ Worcester, MA 01609, USA \\ djamasbi@wpi.edu \\ ${ }^{2}$ Fidelity Investments, User Experience Group \\ 82 Devonshire Street, V3B, Boston, MA 02109, USA
}

\begin{abstract}
Empirical evidence suggests that users often exhibit a viewing pattern that favors the top and left sides of web pages. This viewing pattern may cause users to miss a great deal of information. Grounded in the model of visual hierarchy, this study examines the impact of visual complexity on how users view a page. The results show that users' viewing pattern in our study was more scattered than those reported in previous studies, which used pages with a relatively less complex visual hierarchy. We also examined the impact of browsing and information retrieval on viewing pattern. Such an investigation can provide insight for the design of homepages that can effectively serve both those who browse and those who retrieve information. The results also show that eye tracker can serve as a valuable tool for designers to develop and test new designs.
\end{abstract}

Keywords: Eye Tracking, Design Science, Visual Hierarchy, Viewing Pattern, Fixations, Home Pages.

\section{Introduction}

The Internet has become a valuable source of information for users and a valuable means of communication for companies $[1,2]$. Not surprisingly, providing a positive user experience has become a major focus of industry research [3-6]. One way to improve users' web experience is by enhancing the effective communication of key information on a page. Understanding users' viewing pattern can play an essential role in achieving this goal.

According to Nielsen/Norman research group (http://www.nngroup.com/), people tend to exhibit $F$ shaped reading patterns when viewing a page [7]. The $F$ shaped viewing pattern implies that users are likely to miss key information that is not placed on the left or top of a text based page [7-9]. Users missing information on the bottom of a page is particularly evident in studies showing that users focus more on information above the fold, or the part of page that is visible to users without scrolling $[10,11]$.

Despite the important implications of the F shaped viewing pattern, little work has been done to investigate factors that can affect such a pattern of viewing. Studies have shown that users consistently exhibit an F-pattern of viewing on text-based web 
pages, regardless of whether they are browsing or completing tasks, or whether the text is columnar [12, 13]. However, on purely image-based pages, users do not exhibit the F-pattern [13]. Web pages, and particularly homepages, commonly have a mixture of text and images; viewing pattern on homepages is also of particular importance to firms because research shows that a user's impression of a company can be significantly influenced by the design of the company's homepage [14]. Therefore, this current study expands on previous research by investigating two factors that may affect the F shaped viewing pattern: 1) visual hierarchy, or the arrangement of elements, and 2) task. To conduct these investigations, four prototypes of the homepage from a real company's website were used. Each prototype was manipulated to have a different visual hierarchy. We investigated the impact of two types of tasks, browsing and searching for specific information, on viewing behavior. Because of their relevance to homepages these two types of tasks are often used in usability research [e.g., 10, 13].

\section{Visual Hierarchy}

According to the model of visual hierarchy, viewing pattern is guided by two distinct cognitive processes: searching and scanning. The first phase, searching, refers to a viewer's attempt to find a point of entry into the page. The second phase refers to a viewer's behavior after finding such an entry point. In this second phase, the viewer extracts information which is located around the entry point [15]. This model suggests that both processes can be influenced by the attributes of the web components. For example, the search process can be influenced by attributes such as the size, color, location, text style and visual information (images) of components. Similarly, the scan process can be influenced by attributes such as proximity and order of components. When used effectively, these attributes create a visual hierarchy that can guide users in viewing a page. For example, by manipulating the size or location of an item, designers can manipulate the order in which web components are viewed and thus influence the search process. Because larger items draw more attention than smaller items, larger objects on a page will be viewed before smaller ones. Similarly, because people exhibit a top down viewing preference, items located at the top of a page will have priority in the visual hierarchy over other items on the page. Designers can also influence the scanning phase of viewing. Because items in close proximity are perceived as related to each other [16], placing related information around an entry point on a web page can facilitate a more effective scan phase.

\section{F Shaped Pattern of Viewing}

A number of studies show that users exhibit a viewing pattern that is shaped like the letter " $F "[7,8,17]$. An example of such an F shaped pattern of viewing is displayed in Fig. 1. This viewing pattern tends to contain two long horizontal scans and one long vertical scan. The first horizontal scan tends to be longer than the second one $[13,18]$. 

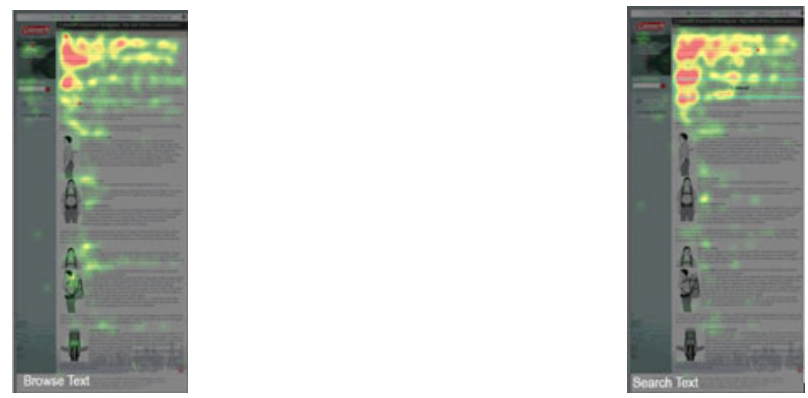

Fig. 1. F-shape pattern of viewing in a text based web page for browsing (the heat map on the left) as well as searching text (the heat map on the right) [13]

According to the above mentioned studies, the $\mathrm{F}$ shaped viewing pattern is not affected by task type, such as browsing or searching (e.g., see Fig. 1). A recent investigation, however, shows that, when using web pages composed primarily of images, users do not show an F shaped pattern of viewing. For example, as shown in Figure 2, the top three rows of images receive similar intense fixations while the bottom two rows receive relatively fewer fixations. Moreover, as shown in Figure 2, the fixation pattern for such a page is not independent of the task. Because images often serve as entry points to web pages [15], these findings suggest that visual hierarchy manipulated by images is likely to have a significant impact on users' pattern of viewing. Moreover, such a visual hierarchy may also affect whether users' viewing pattern is task dependent. The next section contains an explanation of how these possibilities were examined though an eye tracking experiment.
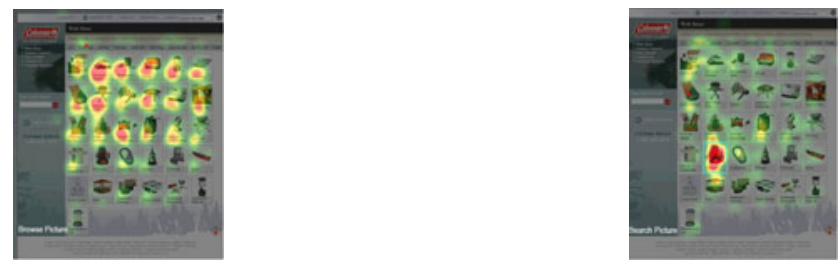

Fig. 2. Viewing pattern for an imaged based webpage browsing (the heat map on the left) as well as searching images (the heat map on the right) [13]

\section{Eye Tracking Experiment}

The theory of visual hierarchy [15] argues that by using size, color, and images, designers can create a visual hierarchy that guides users' attention through a webpage. Such a hierarchy can help users locate entry points and, subsequently, scan for information around those entry points. In other words, according to the theory of visual hierarchy, the design of the page is likely to have an impact on users' viewing pattern. In particular, the visual hierarchy of a page is likely to influence users' tendency to view it in an F-shaped pattern. This viewpoint is 
supported by the findings of previous research [13]. In particular, results suggest that manipulating the visual hierarchy of a page via images may affect users' $F$ shaped viewing pattern [13].

To test the impact of visual hierarchy on $\mathrm{F}$ shape viewing behavior, we used two prototypes of a web page of a real company. These two prototypes were designed to differ in terms of visual hierarchy. One page was designed to have clearer visual hierarchy, with differing font sizes and sectioning (Page A in Fig. 3), while the other was designed to have less clear visual hierarchy, with uniformly sized text (Page B, Fig. 3). The visual hierarchy of each of the two prototypes was further manipulated through the inclusion or exclusion of images of faces. Because images tend to attract users' attention, their inclusion affects the visual hierarchy of the homepage [15]. In this study, images of faces were used because human faces are particularly effective in drawing users' attention [19], and thus can serve as effective points of entry for directing users' attention to information located around them. Based on this, we expected that the inclusion of images of faces on the prototypes to have an impact on users' viewing pattern. In order to examine this, the images of faces were placed in two sections of the page that are typically missed when users exhibit an F shaped viewing pattern: below the fold and on the right side of the page. In the prototype with a clearer visual hierarchy, the faces were placed below the fold of the page, and in the prototype with a less clear visual hierarchy, faces were placed on the right side of the page. The inclusion/exclusion of images of faces on the two prototypes (page A and page B) resulted in a total of four prototypes: two prototypes with clearer visual hierarchy, with and without faces, and two prototypes with less clear visual hierarchy, with and without faces (Fig. 3).

Because task type was of interest in this study, participants were instructed either to browse or to complete an information retrieval task. Because images of faces attract users' attention [5] and serve as points of entry for information that is located in their close proximity [15], their presence on the page may play an important role in users' viewing pattern, both when they browse a page and when they retrieve related information that is placed next to those images.

\subsection{Participants and Design}

Forty eight (33\% male, $67 \%$ female) participants in this study were the employees from various industry sectors such as insurance, finance, real estate, and construction. The age of participants ranged from twenty to fifty years. Participants were randomly assigned to one of the two task conditions, browse or information retrieval. Each participant was also randomly assigned to one of the four prototypes (Fig. 3).

\subsection{Task}

The participants in the task condition were required to retrieve different specific information in random order. For example, the participants were asked to find "an opinion on what's next for GE". Two of these specific pieces of information on the page were located next to images of the faces. 


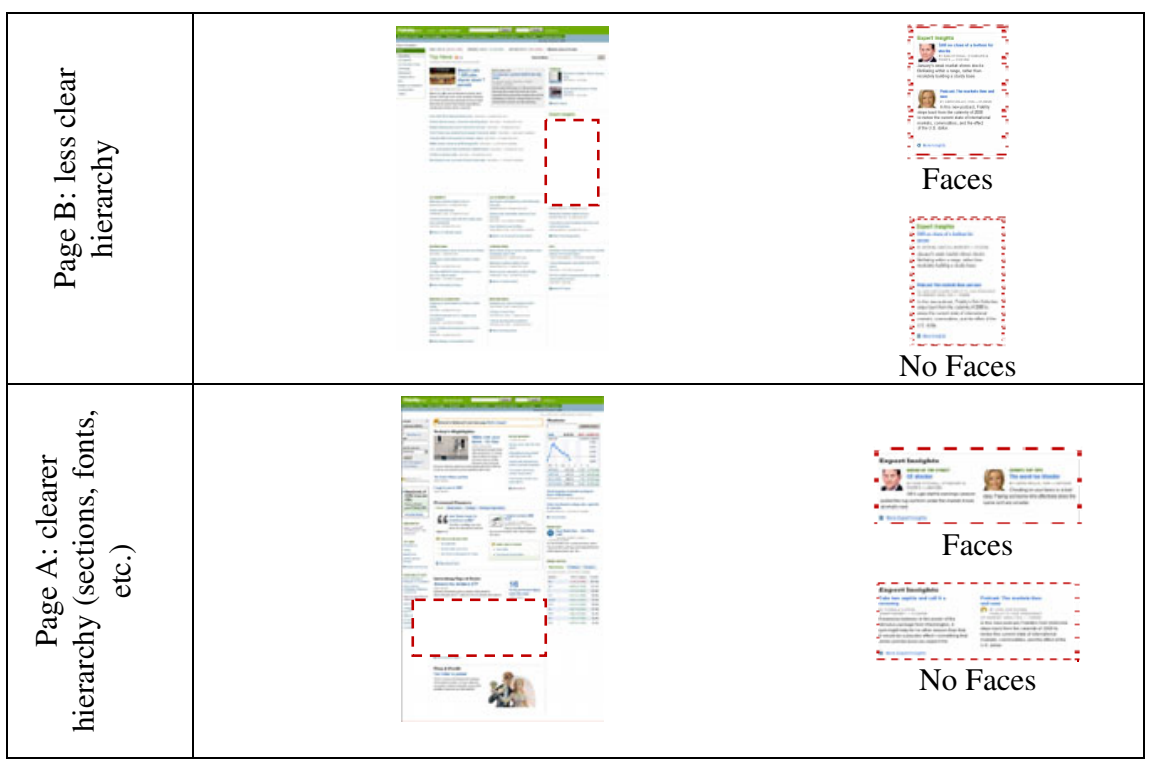

Fig. 3. The prototypes used in our study. The dotted areas show the area where the faces or their textual counterparts were included on the two home pages A and B.

\subsection{Measurements}

Using the eye tracker, the eye movements and fixations of the participants were tracked. A fixation is defined as a gaze of a minimum of 300 milliseconds and reliably indicates interest [20-22]. Fixation patterns are often analyzed using heat maps, which show how long participants fixated on any given area. Heat maps are typically created using data from several participants, allowing the analysis of fixation trends. Examples of heat maps are shown in Fig. 4. Areas with color were fixated upon, with red indicating longer fixations, and green and yellow representing decreasing lengths of fixation. Areas with no color are those that that did not receive fixations.

\subsection{Results}

The analysis of the heat maps (Figure 4) indicate that, regardless of experimental condition (task vs. browsing) and prototype (Page A and B with or without faces), users fixated more above the fold of the webpage. This behavior is consistent with the top-down viewing preference suggested by the model of visual hierarchy [15], as well as the $\mathrm{F}$ shaped viewing pattern $[8,17]$. The fixations in all eight conditions in this study, however, appear more scattered, covering more areas of the page, than the fixations on the heat map from previous studies, shown in Figures 1 and 2. For example, there is much more fixation on the left side of the page, particularly in the information retrieval (search) condition in our study, compared to those shown in Fig. 1 and 2. 


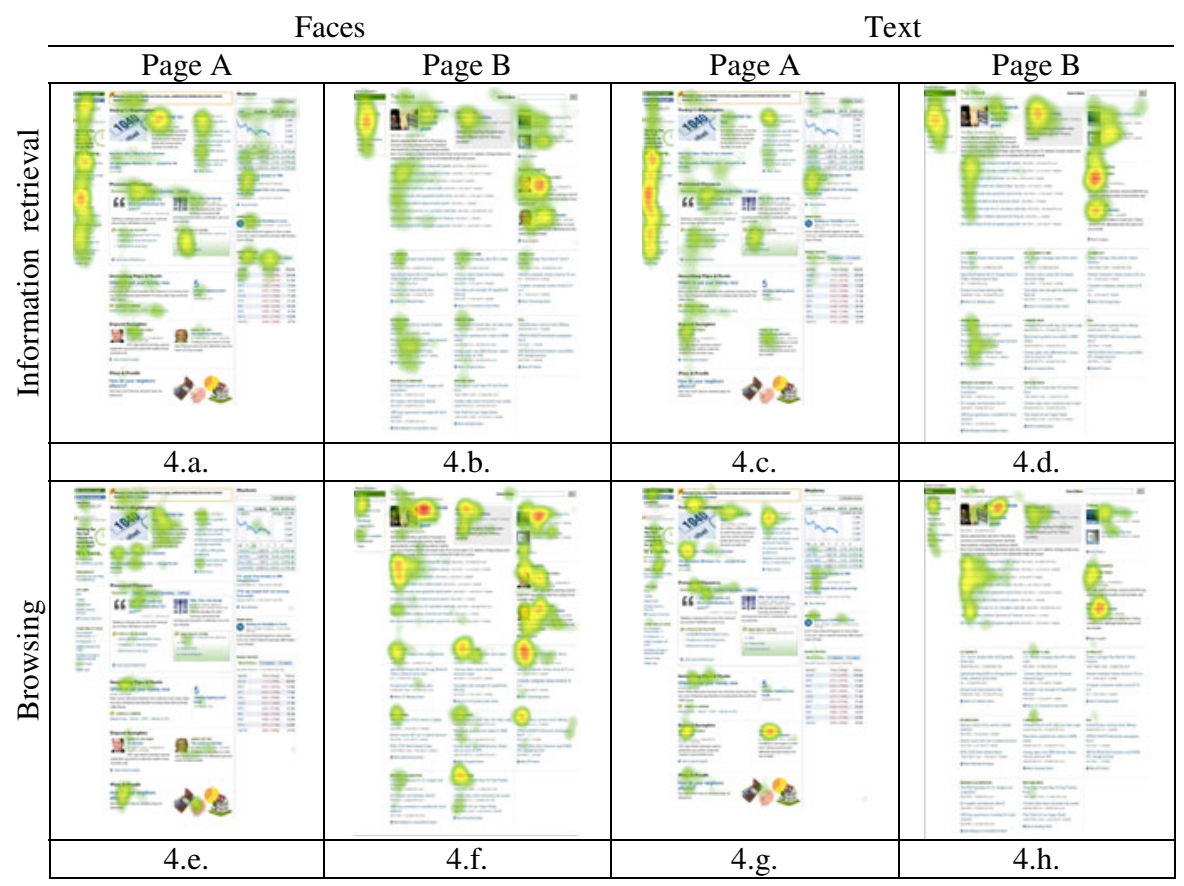

Fig. 4. Heat maps

Comparison of the prototypes provides interesting insight as well. The heat maps for prototypes that did not include faces $(4 \mathrm{c}, 4 \mathrm{~d}, 4 \mathrm{~g}$, and $4 \mathrm{~h})$ show that, regardless of task condition, Page A received more fixation than Page B. This suggests that the clearer visual hierarchy of Page A retained more attention. Additionally, regardless of task condition, Page A received more fixation below the fold. This is particularly important because the area below the fold often receives little attention from users.

The differences between the pages that did not include faces were not evident in the heat maps for the pages that included faces $(4 a, 4 b, 4 e$, and $4 \mathrm{f})$. In the face condition, Page A contained images of faces below the fold, while in Page B the images were placed above the fold. This suggests that changing the visual hierarchy of Page B by placing faces above the fold had a major impact in attracting users' attention. This is particularly evident in Fig. 4f. The fixation pattern of users in Figure 4f shows that the presence of faces above the fold enticed users to browse the page more meticulously, even below the fold.

The comparison of the task conditions also provides interesting insight. In the browsing condition, the fixations appear more in the central part of the page, as opposed to the left or right sections. This is particularly evident in the Page A prototypes that have clearer left, center, and right sections. Consistent with the principle of visual hierarchy [15], the bright yellow on the text around the image on top left corner of the center section suggests that viewers used this image as an entry point when viewing that part of the page. In the information retrieval condition, in contrast to the browsing condition, there was a high amount of fixation on the left side 
of page, where navigational links are located (for example, compare Fig. 4a and 4e). There was also a higher amount of fixation on the right side of the page in the information retrieval condition than in the browsing condition. In other words, the information retrieval condition has a more scattered viewing pattern than the browsing condition. These heat maps together suggest that placing information that is important in retaining new visitors in the center of the page is likely to help attract those who browse a homepage. Placing useful links on the left side of a page is likely to be particularly helpful to those who retrieve information on a page.

In the browsing condition, there was a difference in fixation between the prototypes that included faces and those that did not include faces. This was different, however, depending on the location of the faces. Whether participants were browsing or retrieving information, they spent more time fixating on the manipulated section of the page (the Expert Insight section). When faces were above the fold (4f) they attracted longer fixations on the Expert Insight area than when they were not present (4h). The fixations on the prototype with faces (4f) covered more text around the image compared to the prototype without faces (4h), which shows intense fixation only on the title. When faces were placed below the fold, however, they did not seem to attract a great deal of attention. For example, the heat maps in images $4 \mathrm{e}$ and $4 \mathrm{~g}$ show that the Expert Insights section received more intense fixation when faces were not present. Additionally, it is interesting to pay attention to fixations on faces between the two task conditions. When users were browsing rather than were searching for information, faces received more intense fixations. For example, there is more intense fixation on faces in Fig. 4f, as evidenced by a strong shade of yellow on the faces, compared to Fig. 4b, which has green on faces.

\section{Discussion}

The results showed that the pattern of viewing in this study was different from the pattern shown in a previous study (Fig. 1 and 2). We used prototypes of a homepage that had a more complex visual hierarchy than the previous study. For example, the text in our prototypes utilizes a greater number of attributes (such as size, style, and color) than the text used in Figure 1. The web page used in our study also had fewer images than the page in Figure 2. The combination of text and images, as well as the use of size, color, and location in the main section of our web page creates a more complex hierarchy than the main section of the page in Fig. 2, which contains five rows of equal size images. Thus, the discrepancy between our heat maps and those from previous study indicates that the complexity of visual hierarchy on a page can affect the viewing pattern and guide users to view the page more carefully.

The comparison between the heat maps for the two task types (browsing, information retrieval) in this study showed that the participants concentrated on the central part of the page when browsing, evidenced by shorter fixation on the left or right side of the page compared to fixation on the middle section of the page. When retrieving information, however, the left side of the page (where the links were located) was heavily fixated upon. Moreover, there was higher fixation on the right side of the page (compared to the browsing condition) as well. These results suggest that viewing patterns on the prototypes used in this study were task dependent. 
Because the page in this study had a more complex hierarchy than that of the page used in Fig. 1 and 2, the results of this study indicate that visual complexity may affect the effect of task on viewing pattern.

The comparison between the prototypes also showed that the inclusion of images had an impact on viewing pattern. The results show that the images of faces that were placed above the fold attracted the attention of those who browsed the page to text around them. These images also encouraged users to view the page, even below the fold, more carefully. On the prototype without images of faces, viewers fixated more intensely on the headings as opposed to the text beneath. When the faces were placed below the fold they did not attract as much attention as when they were placed above the fold. For those who were searching for information that was located next to the images of faces below the fold, the fixations were less intense on titles when images of faces were available on the prototype compared to when they were not included on the prototype.

These results have important theoretical and practical implications. Studies show that the visual appeal of a page has a significant impact on users' perception of usability [23, 24]. Visual aesthetics is also shown to be a strong predictor of users' willingness to revisit a page $[25,26]$, suggesting that this work has practical implications for improving the likelihood that website visitors become customers. Because our results show that people fixate more on the center of the page, they suggest that creating visual appeal in this area may be of great importance in creating a favorable aesthetic experience for a user who is browsing a page. These results also contribute to literature by showing that task has an effect on viewing a page with a more complex visual hierarchy. While the participants assigned to browsing concentrated more on the center, those who were tasked with retrieving information focused heavily on the left side of a page. Moreover, this finding is different from previous studies that report a task independent behavior for text-based pages (Fig. 1). More concentration on the left side is also different from the findings of previous studies that show little fixations on the left section of text or image based pages with less complex visual hierarchy (Fig. 1 and 2).

The results indicate that images of faces can serve as an effective entry point to a page. When placed above the fold, these images encouraged users to more carefully inspect the page. When placed below the fold, faces drew the attention of those who browsed the page to the text around them. Additionally, faces attracted stronger fixations when users were browsing the pages. These results suggest that images of faces may play an important role in influencing users' viewing behavior, particularly for those who browse a page. The above results together suggests that eye tracking can serve as an effective tool in developing and evaluating design and thus the analysis of eye tracking data can contribute to human computer interaction research as well as design science.

\section{Limitations and Future Research}

As with any study, the results of this work are limited to its setting. To minimize the effects of the setting the laboratory was designed to create a natural user setting and the eye tracker used was completely remote from participants, allowing for free 
movement. Future studies can expand upon this research by including a prototype that replicates web pages in Figure 1 and 2 to more directly compare the effect of visual hierarchy on F-shaped viewing behavior reported in previous studies. Future studies can also extend our results by examining different genres and types of websites. For example, a retail homepage has different goals than a financial homepage and, therefore, is naturally arranged differently. As a result, these two different types of pages can result in different viewing patterns.

\section{Conclusion}

This research indicates that the $\mathrm{F}$ shaped viewing pattern is affected by the visual complexity of a webpage, and that the type of task does have an effect on the way users view a more complex homepage. These results are consistent with the theory of visual hierarchy and show that a more complex visual hierarchy can guide users' attention to areas outside of the $\mathrm{F}$ shaped pattern of viewing found on less visually complex pages. These results have both theoretical and practical implications. On a broad level, theoretically, this research contributes to design science and human computer interaction research by providing insight about users' fixation patterns when browsing and when retrieving information from a homepage. More specifically, this research expands prior findings on the effect of task on user behavior, as well as the effect of images and, more specifically, images of faces. Practically, this work provides additional understanding of how users view pages. This allows companies to provide a better user experience, which in turn can help attract more users to their websites.

\section{References}

1. Goodwin, B.: Rush to Make the Most of Web 2.0 Sends Developers' Salaries Soaring. Computer Weekly, 48 (2006)

2. Hammer, M., Champy, J.: Reengineering the Corporation: A Manifesto for Business Evolution. Harper Business, New York (1993)

3. Djamasbi, S., Tullis, T., Hsu, J., Mazuera, E., Osberg, K., Bosch, J.: Gender Preferences in Web Design: Usability Testing through Eye Tracking. In: Proceedings of the 13th Americas Conference on Information Systems, Keystone, Colorado, 1-8 (2007)

4. Djamasbi, S., Tullis, T., Girouard, M., Hebner, M., Krol, J., Terranova, M.: Web Accessibility for Visually Impaired Users: Extending the Technology Acceptance Model (TAM). In: Proceedings of the Twelfth Americas Conference on Information Systems, Acapulco, Mexico, pp. 3029-3034 (2006)

5. Djamasbi, S., Tullis, T., Siegel, M., Ng, F., Capozzo, D., Groezinger, R.: Generation Y \& Web Design: Usability Testing through Eye Tracking. In: Proceedings of the Fourteenth Americas Conference on Information Systems, Toronto, Canada, pp. 1-11 (2008)

6. Tullis, T., Siegel, M., Sun, E.: Are people drawn to faces on webpages? In: Proceedings of the 27th International Conference Extended Abstracts on Human Factors in Computing Systems, pp. 4207-4212 (2009)

7. Nielsen, J.: F-Shaped Pattern For Reading Web Content (2006), http://www.useit.com/alertbox/reading_pattern.html 
8. Shrestha, S., Lenz, K.: Eye Gaze Patterns while Searching vs. Browsing a Website. Usability News 9 (2007)

9. Shrestha, S., Owens, J.W.: Eye Movement Patterns on Single and Dual-Column Web Pages. Usability News 10, 1-7 (2008)

10. Buscher, G., Cutrell, E., Morris, M.R.: What Do You See When You're Surfing? Using EyeTracking to Predict Salient Regions of Web Pages. In: Proceedings of the 27th International Conference on Human Factors in Computing Systems, pp. 21-30 (2009)

11. Bailey, R., Koyani, S., Nall, J.: Usability testing of several health information Web sites Technical Report. National Cancer Institute, 7-8 (2000)

12. Shrestha, S., Owens, J., Chaparro, B.: The Effect of Location and Congruency of Text Ads on Information Search. In: Human Factors and Ergonomics Society Annual Meeting Proceedings, vol. 53, pp. 1262-1266 (2009)

13. Shrestha, S., Lenz, K.: Gaze Patterns while Searching vs. Browsing a Website. Usability News 9, 1-9 (2007)

14. Oh, J., Fioritob, S.S., Cho, H., Hofackerd, C.F.: Effects of Design Factors on Store Image And Expectation of Merchandise Quality in Web-Based Stores. Journal of Retailing and Consumer Services 15, 237-249 (2008)

15. Faraday, P.: Visually Critiquing Web Pages. In: Proceedings of the 6th Conference on Human Factors and the Web, Austin, Texas, pp. 1-13 (2000)

16. Flieder, K., Mödritscher, F.: Foundations of a pattern language based on Gestalt principles. In: CHI 2006, Extended Abstracts on Human Factors in Computing Systems, pp. 773-778 (2006)

17. Wilkinson, S., Payne, S.: Eye tracking to identify strategies used by readers seeking information from on-line texts. In: Proceedings of the 13th Eurpoean Conference on Cognitive Ergonomics: Trust and Control in Complex Socio-Technical Systems, pp. 115116 (2006)

18. Nielsen, J.: F-Shaped Pattern for Reading Web Content (2006), http: / / www. useit. com

19. Tullis, T., Siegel, M., Sun, E.: Are people drawn to faces on web pages? In: Proceedings of the 27th International Conference Extended Abstracts on Human Factors in Computing Systems, pp. 4207-4212 (2009)

20. Pan, B., Hembrooke, H., Gay, G., Granka, L., Feusner, M., Newman, J.: The Determinants of Web Page Viewing Behavior: An Eye Tracking Study. In: Proceedings of the 2004 Symposium on Eye Tracking Research \& Applications, pp. 147-154 (2004)

21. Chapman, P.: Remembering what we've seen: Predicting recollective experiences from eye movements when viewing everyday scenes. In: Underwood, G. (ed.) Cognitive Processes in Eye Guidance, pp. 237-258. Oxford University Press, UK (2005)

22. Rayner, K., Liversedge, S.P., White, S.J., Vergilino-Perez, D.: Reading disappearing text: cognitive control of eye movements. Psychological Science 14, 385-388 (2003)

23. Lavie, T., Tractinsky, N.: Assessing Dimensions of Perceived Visual Aesthetics of Web Sites. International Journal of Human-Computer Studies 60, 269-298 (2004)

24. Lindgaard, G., Dudek, C.: User satisfaction, aesthetics and usability: Beyond reductionism. In: Proceedings of IFIP 17th World Computer Congress, Montreal, Canada, pp. 231-246 (2002)

25. Loiacono, E.T., Watson, R.T., Goodhue, D.L.: WebQual: An instrument for consumer evaluation of web sites. International Journal of Electronic Commerce 11, 51-87 (2007)

26. Loiacono, E.T., Watson, R.T., Goodhue, D.L.: WebQual: a measure of Web site quality Marketing Theory and Applications (2002) 